\title{
EDITORIAL
}

\section{Reglas de predicción en neumonía adquirida en la comunidad}

\author{
Prediction rules in community \\ acquired pneumonia
}

The determination of site of care is an essential decision in the management of patients with community-acquired pneumonia (CAP). Patients with mild to moderate CAP may be safely treated at home. Instead, those patients with severe pneumonia must be hospitalized to assure an effective treatment. Severity of CAP is associated with mortality that depends both on the patient's frailty and the intensity of lung inflammation. Because there is no single predictor factor to assess prognosis, diverse prediction rules have been developed to establish severity of CAP and guide the decision of site of care. In our country a new prediction rule, derived from hospitalized patients that incorporate simple clinical variables has been developed. However, this rule requires to be validated in the ambulatory setting before its wide spread use is suggested. Prediction rules are objective and relatively accurate models to assess prognosis that may aid clinicians to evaluate patient's risks and to improve hospitalization decisions. Nevertheless, although the prediction rules may guide the initial management of patients with CAP, they are not intended to replace the clinical judgment, which remains as the «art of medicine»(Rev Méd Chile 2004; 132: 1027-30).

(Key Words: Lung diseases; Neumonia, bacterial; Neumonia, community adquired; Prognosis)

L a neumonía adquirida en la comunidad (NAC) es una enfermedad frecuente en nuestro medio, que ocasiona numerosas consultas en el nivel primario de atención y en las unidades de emergencia. A pesar de que disponemos de potentes antibióticos para su tratamiento, continúa manteniendo una elevada mortalidad, especialmente en poblaciones más frágiles o cuando la enfermedad adquiere mayor gravedad.

El proceso de diagnóstico de la NAC es esencialmente clínico y requiere de una importante capacidad de sospecha de parte del médico, puesto que no todos los pacientes presentan la totalidad de sus síntomas clásicos. Una de las decisiones que tienen mayor impacto en el desenlace de la NAC es la selección adecuada del lugar de tratamiento. Los pacientes con bajo riesgo vital y enfermedad leve o moderada pueden ser trata- dos con antibióticos orales, en su hogar. En cambio, aquellos pacientes con factores de riesgo o que presentan gravedad clínica deben ser ingresados a un hospital para asegurar un tratamiento eficaz en conjunto con una adecuada terapia de soporte vital ${ }^{1}$. Diversos estudios han demostrado que la gravedad de la NAC se asocia fuertemente con su mortalidad. A su vez, la mortalidad depende de la fragilidad del paciente (edad avanzada, presencia de enfermedades concomitantes) y de la gravedad de la neumonía misma (intensidad de la respuesta inflamatoria, características propias del agente etiológico).

La evaluación clínica de la NAC es altamente dependiente de la experiencia del médico clínico. Se ha sugerido que la gravedad de la NAC puede ser subestimada ${ }^{2} \mathrm{y}$, además, puede aumentar rápidamente en poco tiempo. Como no existen 
factores pronósticos únicos con suficiente sensibilidad y especificidad, se han desarrollado diferentes sistemas de puntuación y modelos de predicción que permiten al clínico identificar pacientes con NAC de peor pronóstico.

La Sociedad Británica de Tórax (BTS) desarrolló un modelo de predicción basado en parámetros clínicos y de laboratorio simples, que permiten identificar aquellos casos más graves de NAC con un nivel de evidencia satisfactorio ${ }^{3}$. Este modelo clasifica a los pacientes en tres categorías de gravedad y recomienda su manejo ambulatorio, la observación transitoria en el hospital o el ingreso definitivo al hospital, según cada categoría. Además, es una guía de fácil recuerdo (incluye la depresión del nivel de conciencia, uremia, frecuencia respiratoria, presión arterial y edad) y, al asignar un punto a cada variable, es fácil de calcular.

Por el contrario, Fine y cols derivaron y validaron una regla para identificar pacientes con NAC de bajo riesgo y susceptibles de manejo ambulatorio ${ }^{4}$. Esta regla considera la edad, presencia de comorbilidades y elementos clínicos de gravedad y clasifica a los pacientes en cinco grupos de mortalidad creciente. De esta manera, los grupos 1 y 2 pueden ser tratados ambulatoriamente, con relativa seguridad.

Las reglas de predicción son modelos objetivos de pronóstico, relativamente exactos, destinados a ayudar a los clínicos a evaluar los riesgos de los pacientes y a mejorar las decisiones de hospitalización. Sin embargo, presentan limitaciones que deben ser consideradas en el proceso de toma de decisiones.

Primero, al establecer puntos de corte en variables continuas, como la presión arterial o la frecuencia respiratoria, las reglas de predicción asignan el mismo peso a valores cercanos y valores lejanos del punto de corte. Por ejemplo, la existencia de presión arterial diastólica de 58 $\mathrm{mmHg}$ tiene el mismo peso que una presión de 40 $\mathrm{mmHg}$ y una frecuencia respiratoria de 32 por minuto es igual que una polipnea de 48 por minuto, al momento de calcular la puntuación final. En general, estas reglas de decisión funcionan muy bien en casos con valores extremos, que no ofrecen muchas dudas al médico clínico. En cambio, ofrecen menor ayuda, en aquellos casos con valores cercanos a los puntos de corte, donde la decisión de ingreso puede ser más discutible.

En segundo lugar, el valor de la edad como factor independiente de gravedad ha sido cuestionado. Así, en el modelo de la BTS, se establece un punto de corte en 65 años, asignando igual peso a una edad de 67 años que a una polipnea extrema de 45 ciclos por minuto o a una hipotensión arterial severa. A su vez, en la regla de Fine, se asigna un punto por año de edad (se resta diez puntos a las mujeres), lo que otorga un valor exagerado a la edad y requiere que los enfermos más jóvenes presenten alteraciones fisiológicas más graves para una clasificación similar en comparación con los mayores.

Por último, diferentes autores han enfatizado que estas reglas de decisión pueden ayudar al clínico a adoptar una conducta, pero en ningún caso reemplazan el proceso clínico de toma de decisiones, mucho más complejo que un cálculo aritmético.

En este número de la Revista, Saldías y $\operatorname{cols}^{5}$ describen una regla o herramienta de decisión derivada de una cohorte de 455 pacientes adultos inmunocompetentes hospitalizados por NAC en nuestro medio y proponen que sea utilizada en el modelo ambulatorio para evaluar la gravedad del paciente y decidir el lugar de manejo. Así, asignan tres puntos a la presencia de comorbilidades, hipotensión sistólica bajo $90 \mathrm{mmHg}$ y frecuencia respiratoria entre 20 y 35 ciclos por minuto, dos puntos si hay compromiso de conciencia 0 ausencia de fiebre (definida como temperatura $<37,5^{\circ} \mathrm{C}$ ) y 4 puntos si la frecuencia respiratoria es mayor de 35 por minuto. De acuerdo a esta puntuación, cuando clasifican a los pacientes en categoría I (0 a 5 puntos) sugieren manejo ambulatorio, en categoría II (6 y 7 puntos) recomiendan hospitalización abreviada o vigilancia cercana en el sistema ambulatorio, en categoría III (8 y 9 puntos) sugieren manejo en el hospital y cuando el paciente se clasifica en categoría IV ( $>10$ puntos) recomiendan ingreso a una sala de cuidados especiales. La mortalidad de la serie fluctuó entre 0,9\% en categoría I y 35,8\% en categoría IV.

Esta es la primera vez que, a partir de una población de pacientes nacionales, se deriva una regla de esta naturaleza, lo que otorga mérito al trabajo de los autores. Además, las variables 
consideradas son de fácil obtención por el médico clínico, por lo que pueden ser utilizadas en el hogar del enfermo 0 en los consultorios de atención primaria. Por otra parte, estas variables son similares a aquellas incluidas en las reglas de Fine y de la BTS y en las guías de la Sociedad Torácica Norteamericana. Cuando esta herramienta es evaluada según sus características-receptor operador, exhibe un comportamiento tan bueno como las reglas de Fine o BTS.

Con todo, el estudio descrito amerita ciertas consideraciones:

En primer lugar, de los 455 pacientes estudiados, 292 (64\%) tenían más de 65 años, 338 (74\%) tenían al menos una comorbilidad y 11 (24\%) presentaban compromiso de conciencia. Estas características son propias de una población frágil, lo que explica la inclusión de ausencia de fiebre en el modelo multivariado de predicción. Estos enfermos, que consultan habitualmente en los servicios de urgencia de los hospitales, difieren de la mayoría de los pacientes con NAC que se atienden en el medio ambulatorio, donde se propone evaluar la utilidad de la herramienta.

En segundo lugar, es necesario validar esta herramienta en el contexto de pacientes ambulatorios antes de proponer su aplicación. Al momento de la validación, será necesario definir si el criterio de evaluación será la sensibilidad y especificidad del modelo, o bien se evaluará el impacto que tiene en el médico clínico la utilización de la regla propuesta, seleccionando sólo aquellos pacientes que ofrecen dificultades en la toma de decisiones. En este sentido, se debe tener presente que un índice de evaluación de gravedad debe tener como objetivo mejorar la práctica clínica habitual ${ }^{6}$.

Por último, al obtener valores deducidos de los «odds ratio», cada variable tiene puntuaciones diferentes, lo que dificulta su memorización y, por ende, su aplicación en la práctica clínica por los médicos que trabajan en el nivel primario de atención.

Una solución práctica, de cara a toda nueva regla, es probar su comportamiento. Así, si imaginamos a un varón de 24 años, diabético juvenil, confuso, con presión arterial de 70/40, frecuencia respiratoria de 36 por minuto y $39^{\circ} \mathrm{C}$ de tempera- tura, le asignaríamos 11 puntos y clasificaría en categoría IV. No obstante, para evaluar un enfermo con estas características el clínico no debiera necesitar una regla para determinar que está grave y debe ser ingresado prontamente al hospital. En cambio, a un varón de 24 años, sin comorbilidades, consciente, con presión arterial de 70/40, frecuencia respiratoria de 18 por minuto y $39^{\circ} \mathrm{C}$ de temperatura, le asignaríamos 3 puntos y clasificará en categoría I. Resulta criticable que un paciente con estas características clínicas sea enviado a su hogar con antibióticos orales. Por otra parte, si tenemos una mujer de 60 años, diabética usuaria de hipoglicemiantes orales, con presión arterial de 90/70, frecuencia respiratoria de 22 por minuto y $37,2^{\circ} \mathrm{C}$ de temperatura, le asignaríamos 8 puntos y entraría en categoría III. Sin embargo, esta paciente podría ser manejada en el medio ambulatorio con vigilancia cercana y control de su condición metabólica o podría ser ingresada al hospital transitoriamente.

En conclusión, las reglas de decisión pueden orientar el manejo de los pacientes con neumonía, pero en ningún caso están diseñadas para reemplazar el juicio clínico. El razonamiento clínico, que se aprende de forma tutorial en las escuelas de medicina en campos clínicos acreditados, es un proceso complejo que, hasta ahora, no ha podido ser reemplazado por modelos de inteligencia artificial y continúa formando parte del sarte de la medicina»\%

El presente trabajo tiene el mérito de ser pionero en su campo y de considerar aspectos de la epidemiología local de la neumonía para su derivación. No obstante, las guías clínicas y las reglas de predicción deben tomar en cuenta, además, la configuración local de la red asistencial y los deseos de los pacientes respecto a las características y lugar de tratamiento. Indudablemente, es necesaria más investigación clínica en neumonía adquirida en la comunidad, en nuestro país.

\section{Dr. Patricio Jiménez P. \\ Facultad de Medicina \\ Universidad Austral de Chile, Valdivia, Chile. pjimenez@uach.cl}




\section{REFERENCIAS}

1. Niederman MS, Mandell LA, Anzueto A, Bass JB, Broughton BA, Campbell GD et al. Guidelines for the Management of Adults with Community-acquired Pneumonia. Diagnosis, Assessment of Severity, Antimicrobial Therapy, and Prevention. Am J Respir Crit Care Med 2001; 163: 173054.

2. Neill AM, Martin IR, Weir R, Anderson R, ChereshsKY A, Epton MJ ET AL. Community acquired pneumonia: aetiology and usefulness of severity criteria on admission. Thorax 1996; 51: 1010-16.

3. Lim WS, van der Eerden MM, Laing R, Boersma WG, Karalus N, Town GI et al. Defining community acquired pneumonia severity on presentation to hospital: an international derivation and validation study. Thorax 2003; 58: 377-82.

4. Fine MJ, Auble TE, Yealy DM, Hanusa BH, WeissFELD LA, Singer DE et aL. A prediction rule to identify low-risk patients with community-acquired pneumonia. N Engl J Med 1997; 336: 243-50.

5. Saldías F, Farías G, Villarroel L, Valdivia G, Mardónez JM, Díaz A. Diseño de un índice pronóstico clínico para el manejo de la neumonía del adulto adquirida en la comunidad. Rev Méd Chile 2004; 132: 1037-46.

6. Woodhead M. Assessment of illness severity in community acquired pneumonia: a useful new prediction tool? Thorax 2003; 58: 371-2. 